\title{
Air Traffic Controllers' Control Strategies in the Terminal Area under Off- Nominal Conditions
}

\author{
Lynne Martin, Joey Mercer, Todd Callantine, Michael Kupfer \& \\ Christopher Cabrall \\ San Jose State University \\ NASA Ames Research Center \\ Moffett Field, California \\ Lynne.Martin@nasa.gov, Joey.Mercer@nasa.gov, Todd.Callantine@nasa.gov, \\ Michael.Kupfer@nasa.gov, Christopher.D.Cabrall@nasa.gov
}

\begin{abstract}
A human-in-the-loop simulation investigated the robustness of a schedule-based terminal-area air traffic management concept, and its supporting controller tools, to off-nominal events - events that led to situations in which runway arrival schedules required adjustments and controllers could no longer use speed control alone to impose the necessary delays. The main research question was exploratory: to assess whether controllers could safely resolve and control the traffic during off-nominal events. A focus was the role of the supervisor - how he managed the schedules, how he assisted the controllers, what strategies he used, and which combinations of tools he used. Observations and questionnaire responses revealed supervisor strategies for resolving events followed a similar pattern: a standard approach specific to each type of event often resolved to a smooth conclusion. However, due to the range of factors influencing the event (e.g., environmental conditions, aircraft density on the schedule, etc.), sometimes the plan required revision and actions had a wide-ranging effect.
\end{abstract}

Keywords: CMS, ATC, traffic management strategy, collaborative work 


\section{INTRODUCTION}

In today's air traffic system, control in a busy terminal area can include multiple speed changes, altitude level-offs, and/or heading vectors. In periods of heavy demand, this very safe system works hard to maintain high throughput, but does so at the expense of flight efficiency. That efficiency is commonly traded for positive control, so that controllers can quickly deliver aircraft from one sector to the next with proper spacing that allows aircraft to safely descend for landing. When demand is low, there is more space between aircraft and therefore less reason to be concerned with compression; less controller intervention is needed and aircraft can more easily descend along an efficient descent profile.

The Super-Density Operations research under NASA's Airspace Systems Program aims to safely sustain high runway throughput while still accommodating fuel-efficient operations. Advanced scheduling capabilities create schedules at the runway to enable Optimized Profile Descents (OPDs) along Area Navigation (RNAV) routes. Assuming en route controllers feed the Terminal Radar Approach Control (TRACON) with reasonable schedule errors, TRACON controllers can avoid costly altitude and heading maneuvers and instead rely primarily on speed adjustments to minimize runway schedule conformance errors (Isaacson, Robinson, Swenson, \& Denery, 2010).

Controller-Managed Spacing (CMS) research in the Airspace Operations Laboratory (AOL; Prevôt, et al., 2010) has used a series of real-time human-in-theloop simulations to investigate specific controller decision support tools (DSTs) for such operations. With relatively straight-forward display enhancements, TRACON controllers were able to manage dense arrival flows that followed OPDs along RNAV routes and met runway schedule times (see Kupfer, Callantine, Martin, Mercer \& Palmer, 2011; Callantine, Palmer \& Kupfer, 2010).

Having tested the CMS concept and tools under conditions with only speed variances, in 2011 research examined how robust the concept was to significant disturbances and off-nominal conditions. Accepting that large disturbances to

operations in the TRACON are an eventuality, the response and recovery to an offnominal event also requires investigation. In particular, the research sought to investigate whether the CMS concept and tools can support the response to and recovery from a disturbance (see Callantine, Cabrall, Kupfer, Martin, Mercer \& Palmer, 2011).

This paper will focus on the role of the supervisor, who managed the runway schedule as part of the controller team's response to scripted off-nominal events. The paper will describe in more detail the operations tested, the CMS tools, and the roles and responsibilities of the participants.

\section{METHODS}

The 2011 simulation built on the previous studies using some of the same elements (e.g., the airspace), extending the investigation of other elements (e.g., 


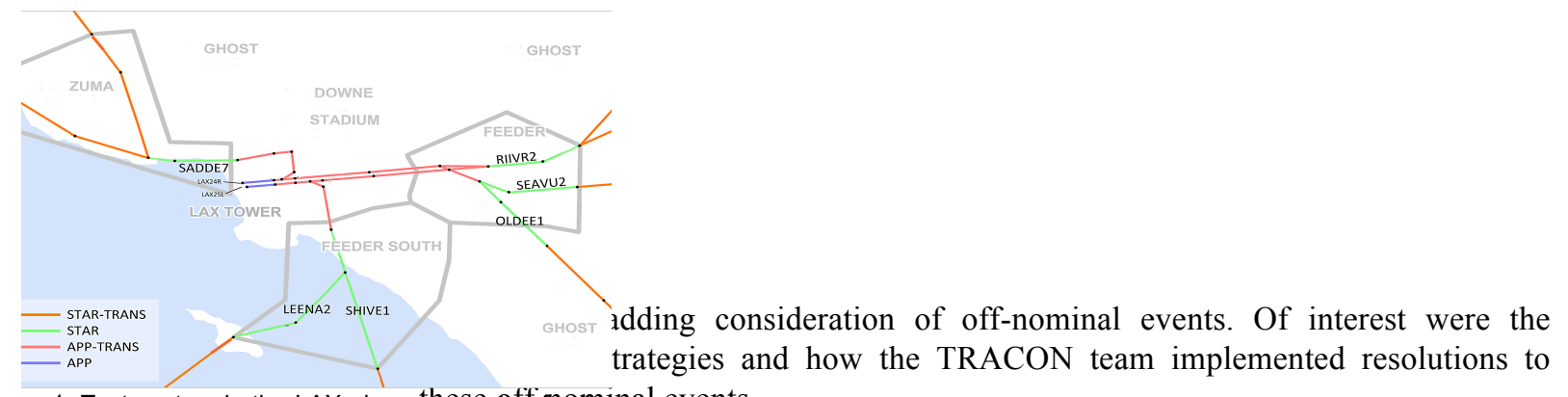
re 1. Test sectors in the LAX airspatebeatedforimenal events.

Ilation

\subsection{The Simulation: Route Structure, Scenarios and Winds}

The airspace simulated was the terminal area around the Los Angeles International Airport (LAX). Figure 1 shows a map of the airspace displaying the routes, waypoints, and sector boundaries for west-flow operations (based on current sectors in the Southern California TRACON). The airspace was comprised of three feeder sectors: Zuma, Feeder and Feeder South, and two final sectors: Stadium and Downe. Aircraft in the simulation flew OPDs on merging RNAV routes to runways LAX24R and LAX25L. Path options in the form of named RNAV

arrival/approach transitions were made available inside the TRACON to absorb large delays. RNAV go-around routes were designed to enable controllers to use the CMS tools to reinsert go-arounds into the arrival flows to the runways.

Three one-hour scenarios were developed for the simulation with a planned mean throughput of 31 arrivals to each runway. The scenarios were built under the assumption that aircraft had been delivered to the TRACON meter fixes by en route control with nominal schedule errors between 60s early and 30s late. However, due to wind forecast errors, these errors between the estimated time of arrival (ETA) and scheduled time of arrival (STA) at TRACON entry differed from that range. In addition to the standard wake spacing distances an additional buffer of $0.5 \mathrm{nmi}$ (Nautical Miles) was added into the scheduler.

Winds were a headwind aligned with the landing runways from $265^{\circ}$ a third of the time, and the rest of the time were from $45^{\circ}$ north or south of the runway. Below $1,500 \mathrm{ft}$ the forecast wind profile matched the actual wind profile, but above this altitude there were two wind-forecast-error conditions where the actual wind differed from the forecast wind by either 7 or 13 knots. This had an effect on the accuracy of the higher-level tools (see below) because their calculations took the forecast winds into account.

Four types of off-nominal event were planned: on-board medical emergencies, radio outages (NORDO or "no radio"), pilot-initiated go-arounds (e.g., due to gear malfunctions), and tower-initiated go-arounds (e.g., due to another aircraft on the runway). Two of these events, of different types, were scheduled in each run.

The study was run in the Airspace Operations Laboratory (AOL) at the NASA Ames Research Center using Multi Aircraft Control System (MACS) software 


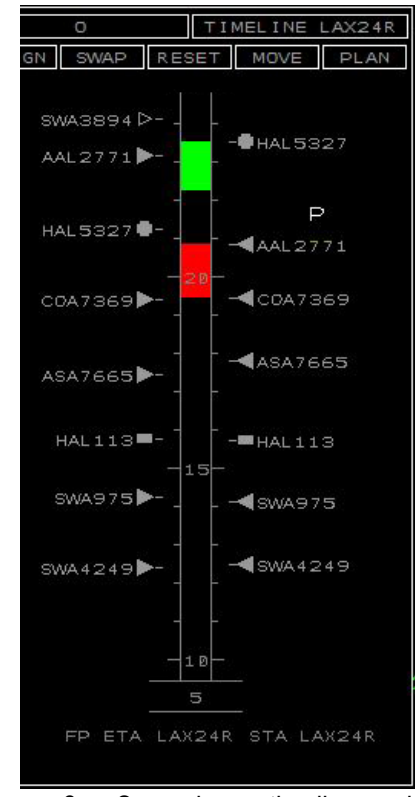

$\supseteq 2$. Supervisor timeline with Juling tools
(Prevôt, et al., 2010). Simulated aircraft were assumed to be Flight Management System and Automatic Dependent Surveillance-Broadcast-out equipped.

\section{$2.2 \quad$ CMS Tools}

Controller participants worked with a MACS emulation of the Standard Terminal Area Replacement System (STARS) onto which the CMS tools were added. As the Supervisor's role was to manage the traffic schedule, his four tools were located on two timelines (Figure 2). The two timelines showed schedules to the two arrival runways with aircraft ETAs on the left side of the time tape and their STAs on the right. The ETA computations and the tools were based on aircraft trajectories being predicted through the forecast winds. Green bars in the time tape indicated excess spacing, or "gaps," in the schedule and red bars indicated insufficient spacing (overlap). The Supervisor's four tools were located in a row of buttons near the top of each timeline. He was able to "assign" a particular STA to an aircraft by dragging its current STA to a new desired time; "swap" the STAs of two aircraft, i.e., the STA of aircraft $b$ became the STA of aircraft a and vice versa; "reset" all aircraft after a specified time which re-scheduled aircraft according to the current ETA information; and, "move" multiple aircraft STAs forward or backward on the schedule by a constant amount. These tools are based on aircraft trajectory predictions computed using the forecast winds. The timelines were located on a display ranged out to show the entire TRACON as well as about $40 \mathrm{nmi}$ into the Center airspace (approx 100nmi radius).

The controllers also had a suite of four tools. These differ from the Supervisor's tools as they are focused on individual aircraft rather than the schedule. The CMS controller tools are designed to provide a temporal and spatial awareness of each aircraft's progress relative to its STA, and speeds a controller could issue to correct schedule errors (see Kupfer, et al., 2011 for a detailed description).

\subsection{Participants and Their Tasks}

Eight retired air traffic controllers participated in this simulation. Three staffed the feeder positions and two the final positions. The sixth participant, who served as the Supervisor, was recruited specifically for his professional experience as a terminal-area traffic manager. Two controllers staffed supporting confederate 
"ghost" and tower positions, and general aviation students and pilots ran eight simulation pilot stations to control the simulated aircraft.

Controllers were asked to manipulate the traffic using only speed and the predefined path options, if possible, to bring the aircraft to land at the runways on schedule, although vectoring was still a valid option. The Supervisor was asked to manage the schedule and to try to maintain high runway utilization by working the schedule only as far out as the first aircraft that was outside the freeze-horizon (about $80 \mathrm{nmi}$ out from the runways). How the controllers and the supervisor used the tools to achieve this and how they coordinated to formulate off-nominal recovery plans was a point of research interested and left open for them to determine.

Participants took part in five days of training during shakedowns prior to the study to ensure their familiarity with the concept, tools, and procedures. The study ran for two weeks (non-consecutive) during which 46 one-hour runs took place -42 runs were unique and four were repeats. Each run had two scripted off-nominal events that occurred in three base scenarios under varying wind conditions. The run matrix was randomized to reduce controller learning effects. All but one scripted event initiated as planned, yielding 91 off-nominal event examples.

Data were recorded for each run through the MACS' data collection logs, including aircraft and tool states, as well as controller and pilot actions. Screen and voice recordings were also collected. Following each run, the participants completed an online questionnaire that included questions about their strategies and problem solving. In addition, detailed observer notes were compiled from each experimental run and debriefing. The following section describes results from analyses conducted thus far.

\section{RESULTS}

The Supervisor's role was to manage the schedule manipulations that were required during off nominal events. As this was an exploratory position, the aim of the study was to observe the strategies the Supervisor developed and how they were executed, and to record his interactions with the rest of the team, who were controlling the traffic in the TRACON.

\subsection{Supervisor Strategies}

The Supervisor developed four basic strategies that he often used to begin handling the four different types of off nominal event. When a NORDO aircraft was identified, the Supervisor typically began by setting its STA to match its ETA (using his "assign" tool). He then assessed the schedule and how aircraft ahead or behind the NORDO aircraft could be affected. He usually consulted with the feeder controller(s) to determine whether to swap STAs, and in some cases devised a contingency plan for having the aircraft ahead of the NORDO go around if safe spacing was lost. For medical emergencies, the Supervisor coordinated with the controllers to expedite the emergency aircraft if possible; the plan could include schedule swaps and delaying other aircraft. When go-arounds were declared, the controllers needed to formulate a plan for climbing the aircraft to a safe altitude and assigning the desired RNAV route to re-enter the aircraft into the flow. The 
Supervisor informed this route choice because he considered the schedule and adjustments of neighboring aircraft in the planned sequence. In some cases, the Supervisor organized the aircraft to go around to the other arrival runway if its schedule had more space. For tower-initiated go-arounds, less time was spent developing a plan because the event was announced much later on an aircraft's final approach than for pilot-initiated go-arounds.

These differences in strategies were reflected in the work that the Supervisor estimated he did for each type of off-nominal event (Table 1). For example, he worked three times as long, and twice as hard, on NORDO problems as on medical events because for medical events he was able to set up a plan and let it play out, whereas in a NORDO event the actions of the aircraft were unknown, even though they were expected to follow the charted procedure, which resulted in NORDOs entailing higher levels of monitoring.

Table 1. Supervisor's workload during four types of off-nominal events

\begin{tabular}{|l|c|c|c|c|}
\hline & $\begin{array}{l}\text { Medical } \\
\text { Emergency }\end{array}$ & $\begin{array}{l}\text { Pilot initiated } \\
\text { go-around }\end{array}$ & $\begin{array}{l}\text { Tower initiated } \\
\text { go-around }\end{array}$ & NORDO \\
\hline Mean estimated task time & 2 min 46 s & 5 min 15 s & 5 min 15 s & 9 min \\
\hline $\begin{array}{l}\text { Mean estimated task mental } \\
\text { load }\end{array}$ & 1.76 & 2.87 & 2.9 & 3.62 \\
\hline $\begin{array}{l}\text { Mean estimated task time } \\
\text { pressure }\end{array}$ & 1.66 & 2.41 & 2.85 & 3 \\
\hline
\end{tabular}

\subsection{Variations to a Plan}

Unsurprisingly, not every strategy played out the way the Supervisor intended. Some plans fell victim to wind changes or to other actions that impacted them. For example, in Trial 25 the wind shift to a direct headwind pushed the ETAs of all aircraft back, making them late with respect to the schedule; while in Trial 45 a favorable wind change helped an aircraft advance into its slot marker at an earlier waypoint. Some strategies simply followed a different course than the one the Supervisor predicted. For example, in Trial 36 a NORDO aircraft kept its speed up, forcing the Supervisor to abandon his plan of schedule 'tweaks' and take an aircraft around to the other runway.

The Supervisor reported he made adjustments to his original plan about $60 \%$ of the time but these were most often 'a few adjustments' and were 'large revisions' for less than $10 \%$ of the events. Usually he did not adjust a medical event plan (median $=1$ out of 7: no adjustments) but he had to make 'a few adjustments' to plans for NORDO events (median =3). In a companion question, the Supervisor was asked what he would have done differently in hindsight. He reported he would not have done anything differently in $73 \%$ of the events, but he would have made changes on 15 occasions (17\%). Half of these reports were after NORDO events and, although the Supervisor would usually have done more (Figure 3), on one or two occasions he thought a better course of action would have been to do less: "I would have let the NORDO run and monitored the situation," (Trial 36). On four occasions he felt his initial plan would have worked and he should not have made the additional adjustments that he did. For example, in Trial 17, after initially saying 


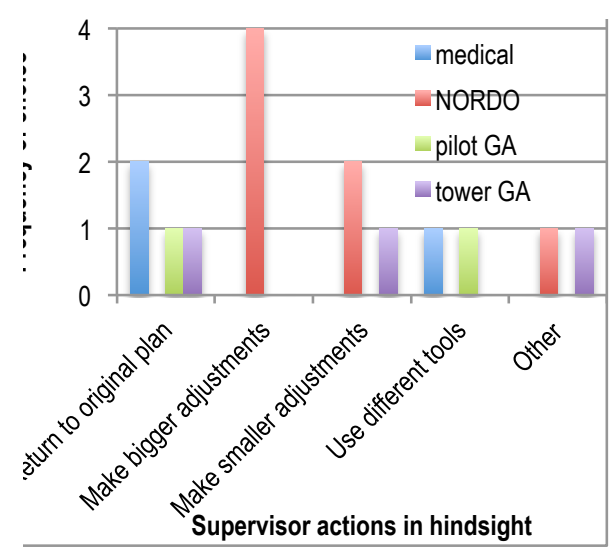

ure 3. Supervisor's reports of different plans in dsight the aircraft with the medical emergency should stay at its assigned STA, the Supervisor decided to swap it with the aircraft in front. To achieve this, Stadium had to issue multiple vectors to both aircraft - a large increase in workload.

The larger number of adjustments and plan changes (with hindsight) that the Supervisor made during the NORDO events highlight that the NORDOs, in general, were the least flexible of the four event types. Not only was a NORDO inherently less predictable, because the pilot could not be contacted, it was also less manipulable because the controllers could not redirect the problem aircraft and the Supervisor had to adjust the schedule around it.

\subsection{Collaboration}

As the team-member with an overall view of the TRACON traffic and the schedule, the Supervisor was the natural role to initiate recovery plans for offnominal events. Plans were sometimes formed by the Supervisor alone but often, if there was time, he would discuss the situation with the controllers to generate a plan that was informed by their views. For example, in Trial 29 the Supervisor considered a swap but as part of a discussion with Downe he demonstrated this action and Downe said that he would not be able to meet the new STAs. Due to this, they began to work on other options. This strategy of consulting the team was usually worthwhile because the controllers had to be able to execute the plan that the Supervisor created. However, controllers often did far more than merely "execute" the plan. In approximately a third of the events the plan would not have had the successful outcome that it did if a controller had not paid additional attention to the key aircraft and creatively manipulated them into the right place. Sometimes these actions were to use the path extension routes or extra speed control but at other times the controller solution reflected years of experience; in Trial 28 Downe vectored one aircraft off the final in a U-shaped detour that allowed another aircraft to overtake without having to swap them.

Sometimes a plan was determined or requested by a controller. Although the Supervisor estimated that he generated the plan $60 \%$ of the time (Figure 4 ) and he collaborated $30 \%$ of the time, these were occasions where the controller suggested a distinctly different option (not the creative execution discussed above). Joint plans were created most often for tower-initiated go-arounds probably because the initial actions for this event - to break an aircraft out of its final descent and assign it the 


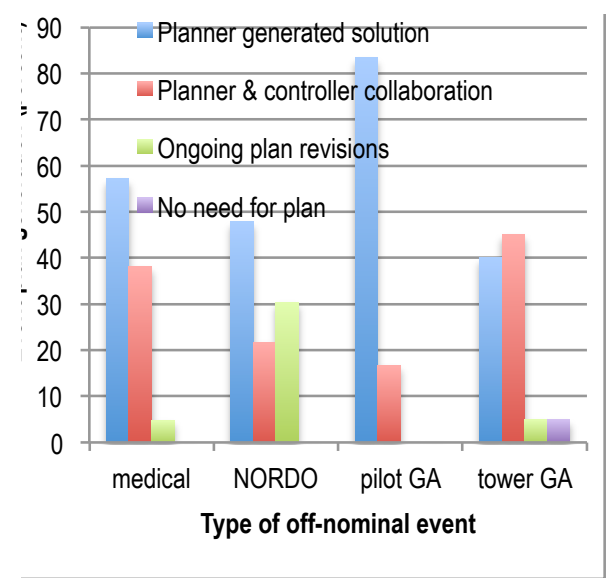

Ire 4. Supervisor's reports of how plans were rated

go-around route - had to happen quickly. Final controllers often assigned a route and then asked the Supervisor if it would work which began an exchange of ideas.

In the handful of cases where there needed to be a new plan controllers were involved half the time in the Supervisor's revisions. This included asking the Supervisor for additional help because the STA could not be met, reporting that a plan would not work for their position, and working through options to find a viable solution.

\subsection{Interactions of actions}

Given that the Supervisor's role was a new component of the research and that he didn't have clearly defined procedures to follow, there was a certain degree of experimentation as his role organically evolved and he became more comfortable. While this was a necessary part of the learning process, it also came with some interesting side effects.

The supervisor tools were all related to adjusting the runway schedule; his actions directly changed the ground system's STA of an aircraft. At the same time however, the controllers' tools were driven by aircraft STAs. Consequently, DSTs on the controller displays fluctuated as a direct result of the supervisor's actions. This was usually a good thing: e.g., an aircraft needed to be delayed to make room for the re-insertion of a go-around aircraft. In such a case the controller tools helped to understand the current state of the aircraft relative to meeting its goal of the new STA. On the other hand, there were situations when formulating a plan was difficult for the Supervisor and required him to "try out" a few potential ideas before finalizing the plan. To do this, the Supervisor simply adjusted the STAs of the affected aircraft in a particular way and examined the result (sometimes with and sometimes without controller feedback). At that point, the supervisor could have kept the plan, reverted to the original state, or tried adjusting the STAs of the affected aircraft in a different manner. Even if a plan formulation like this took less than a minute, during that time the controller DSTs could change dramatically in response to the supervisor's actions. In multiple instances, a controller saw the change to their aircraft's STA and immediately began working toward the new goal, only to discover that it was a "test" rather than the finalized goal.

A more extreme example shows how the interaction of the Supervisor's planning process and the controllers' efforts to manage their aircraft can cause undesired outcomes. In Trial 35, the NORDO aircraft was a little ahead of schedule, and the Supervisor planned to swap the NORDO with the aircraft scheduled just ahead 
(flight HAL5327). After the swap, HAL5327 was ahead of its STA and the Zuma controller working the flight issued it a path extension as well as a speed reduction. Three minutes went by while the Supervisor examined the changes he made to the schedule, after which he determined this plan would not work, and swapped the two aircraft back. This now put HAL5327 behind its STA, so much so that Zuma informed the Supervisor that even with speed increases the STA could not be met. The Supervisor adjusted the schedule a third time, entailing moving the HAL5327 STA back again, as well as delaying several other aircraft STAs. Zuma slowed HAL5327 again, and was somewhat frustrated at having issued multiple contradictory speed clearances. He then spent the rest of Trial 35 absorbing sizable delays for most of the aircraft flying through his sector.

In this example, the Supervisor's delay in solidifying a plan without coordination, combined with the actions already taken by the controllers, complicated the problem unnecessarily, and resulted in an inefficient solution. Interestingly, Trial 35 was repeated as Trial 46, and in Trial 46 the supervisor did not attempt to swap the NORDO aircraft with HAL5327, yielding a much smoother outcome. Figure 5 shows the lateral tracks of aircraft that flew through the Zuma and Stadium sectors during Trials 35 and 46.
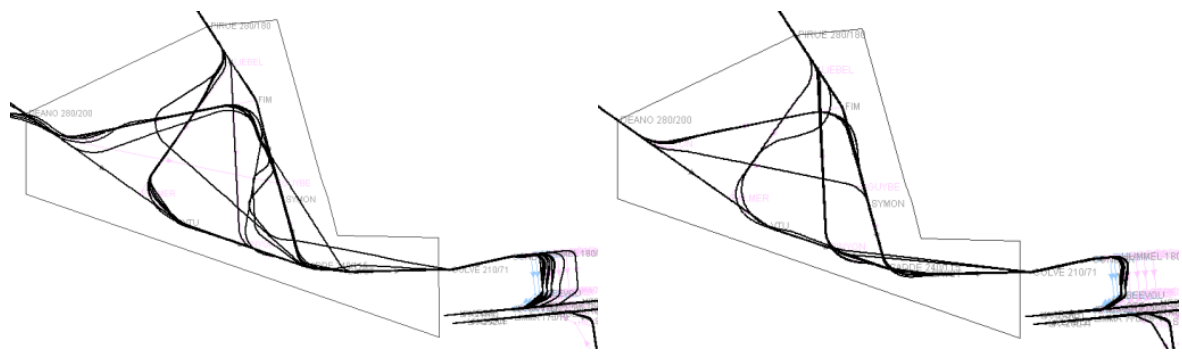

Figure 5. Lateral tracks of aircraft flown through Zuma and Stadium sectors in Trial 35 (left) and Trial 46 (right). Compared to Trial 46, the tracks in Trial 35 show increased vectoring in both the Zuma and Stadium sectors, stemming from the Supervisor's inefficient plan.

\section{$4 \quad$ DISCUSSION}

While prior studies of nominal operations (Kupfer, et al., 2011) illustrated little need for schedule manipulations, this study of off nominal events clearly shows the importance of the role that the Supervisor played. Although his responsibilities were pre-defined as monitoring and managing the schedules, the role of 'strategist' and the procedures for managing events were specifically not defined. During the study, the need for a single coordinator role emerged. As the position with an overview of the TRACON the Supervisor was able to identify options - such as swapping aircraft to a different runway, especially in the go-around cases - that the sector controllers were unable to see. In this sense, he became the team strategist as well as the schedule manager. During the debrief, when asked who should be in charge of creating the plan, the controllers echoed the idea the supervisor was in the best 
position to do so, stating "I knew [the supervisor was] the only one with the big picture. I learned that [lesson] early; that I've got the small picture."

Over the course of the two-week study, the TRACON team developed initial strategies for each of the four event types that gave them a going-in position that they modified to meet the specific conditions of each trial. These strategies could become the basis for developing specific procedural guidance for managing these particular types of off-nominal events. Notwithstanding this, the trials in this study provide case-studies for comparing the different approaches the team tried and illustrate how some approaches were more effective than others at bringing the schedule back to a nominal state. Further research is needed to ensure off-nominal recovery procedures are specified at the correct level of abstraction and conditions for applying particular procedures are clearly defined (Callantine, 2011). It is interesting that although the same basic event was initiated multiple times, they rarely played out in the same way.

The functioning of the controllers and the Supervisor as a team unit were highlighted by the off-nominal events. During nominal operations there was little need for the team to coordinate because the CMS tools provided the required information. However, off-nominal events illustrated that supervisor-controller communication was key to many of the successful solutions the team identified. This is most definitely not a one-way channel; controller input was valuable to the Supervisor in many cases. Not only do the controllers have a detailed view and understanding of the situation that a supervisor does not have but in this study, he needed their feedback to assess whether he had solved a problem or just shifted it. A "ripple" effect was observed for some Supervisor actions where workload increased in sectors that were not involved with the off-nominal problem (usually the East Feeder).

As seemingly small actions on the schedule can have large and often unforeseeable consequences, off-line planning tools are key. Problems were observed that were complicated by the Supervisor trying a solution to look at its effects and reversing his actions but not before the controllers had begun to issue clearances based on the change. The team tried to develop a workaround where the Supervisor announced when he was planning and when he had set the schedule, but this frustrated the controllers as their tools reset and they had to wait to make adjustments. The Supervisor needed a 'schedule trial-planning' function so he could assess the implications of his plans before he set them as changes into the ground automation. A pre-existing MACS schedule-adjustment functionality could be streamlined and extended to enable schedule trial-planning to meet this requirement in future studies. Further analyses to determine whether any of the Supervisor's tools could or should be automated also need to be conducted.

\section{$5 \quad$ CONCLUSION}

This study investigated the robustness of a schedule-based arrival-management concept using controller tools and introduced a new supervisory position to manage the schedule under off-nominal events. The controller team successfully managed most of the trial cases during a two-week study, suggesting that this concept has potential to demonstrate consistently robust performance even during off-nominal events. The results indicate recovery from off-nominal events is most efficient when 
rescheduling is accomplished reasonably quickly and the TRACON team is able to use the tools to make the required adjustments. The larger number of adjustments that the Supervisor made during the NORDO events and the greater number of actions he would have changed with hindsight illustrate that the NORDO events, in general, were the hardest, and most workload-intensive to manage. The case studies have provided rich information about possible strategies for development of offnominal recovery procedures, and have shown where improvements to the supervisor tools are required and where the study can be improved for future investigations. While the research is a first step toward establishing the necessary safety case for CMS operations, the results are promising, and should help pave the way for future development of controller tools, procedures, and simulation-capabilities.

\section{ACKNOWLEDGEMENTS}

Thank you to the AOL development team and the controller subject matter experts without whom this study could not have been completed.

\section{REFERENCES}

Callantine, T. 2011. Modeling off-nominal recovery in NextGen terminal-area operations, AIAA-2011-6537. Proceedings of the AIAA Modeling and Simulation Technologies Conference, Portland, OR.

Callantine, T., C. Cabrall, M. Kupfer, L. Martin, J. Mercer \& E. Palmer. 2011. Investigating the impact of off-nominal events on high-density "green" arrivals. $30^{\text {th }}$ Digital Avionics Systems Conference, October 17-20 ${ }^{\text {th }}$, Seattle, WA.

Callantine, T., E. Palmer \& M. Kupfer. 2010. Human-in-the-loop simulation of trajectory based terminal-area operations. 27 $7^{\text {th }}$ International Congress of the Aeronautical Sciences (ICAS), Nice, France.

Isaacson, D., J. Robinson III, H. Swenson \& D. Denery. 2010. A concept for robust, high density terminal air traffic operations. Proceedings of the AIAA Aviation Technology, Integration, and Operations (ATIO) Conference, Fort Worth, TX.

Kupfer, M., T. Callantine, L. Martin, J. Mercer \& E. Palmer. 2011. Controller support tools for schedule-based terminal-area operations. Proceedings of the Ninth USA/Europe Air Traffic Management Research and Development Seminar, June, Berlin, Germany.

Prevôt, T., P. Lee, T. Callantine, J. Mercer, J. Homola, N. Smith \& E. Palmer. 2010. Humanin-the-loop evaluation of NextGen concepts in the Airspace Operations Laboratory, AIAA 2010-7609. American Institute of Aeronautics and Astronautics, Reston, VA. 